\title{
Velocidade do fluxo e pH salivar após radioter apia da região de cabeça e pescoço
}

\author{
Salivary flow rate and $\mathrm{pH}$ after radiotherapy of the head and neck region
}

\author{
Antonio Adilson Soares de Lima ${ }^{1}$, Maria Antonia Zancanaro de Figueiredo², Stella Maria Rigo Krapf ${ }^{3}$, Fabiany Rodrigues de Souza ${ }^{3}$
}

\begin{abstract}
Resumo
A hipossalivação é uma seqüela provocada pelo tratamento radioterápico das neoplasias malignas da região da cabeça e pescoço e esta condição predispõe o paciente às lesões de cárie e infecções bucais. Este estudo avaliou 0 comportamento do fluxo e pH salivar num grupo de 42 indivíduos submetidos a tratamento radioterápico com dose diária de 200cG y por aproximadamente cinco semanas. Seis amostras de saliva total foram colhidas de maneira padronizada em cada indivíduo. A primeira amostra foi obtida antes do tratamento, a segunda durante e as demais por até seis meses após o tratamento ter sido encerrado. 0 fluxo salivar e o pH foram determinados através de métodos laboratoriais. Ao receberem a dose de $1500 \mathrm{cG}$ y, período que equivaleu ao oitavo dia de tratamento, o fluxo salivar dos indivíduos diminuiu em $50 \%(p \varangle 0,05)$. Ao final do tratamento, a média observada para o fluxo foi de $0,33 \mathrm{ml} / \mathrm{min}$, o que representou uma redução de $79 \%(p<0,05)$. 0 pH da saliva também diminuiu em função do tratamento radioterápico. A média do pH registrada antes do tratamento foi de 7,6 que diminuiu e manteve-se em 6,8 por até seis meses após o tratamento radioterápico $(p<0,05)$. Estes resultados demonstraram que a radioterapia quando aplicada na região da cabeça e do pescoço provoca uma redução progressiva no fluxo e pH salivar em função do tempo.
\end{abstract}

Palavras-chave: Neoplasias de cabeça e pescoço; Xerostomia; pH ; Saliva.

${ }_{1}^{1}$ Professor D outor Titular do Curso de O dontologia da PUCPR

2 Professora D outora Titular do Curso de O dontologia da PUCRS

3 Bioquímica do H ospital São Lucas da PUCRS

Trabalho realizado no curso de $O$ dontologia da PUCPR

Endereço para correspondência: Prof. Dr. A.A.S.L. - Rua João Pontoni 120 apto. 104 Cristo Rei - 80050-490 Curitiba/PR . E-mail: adillima@terra.com.br 


\begin{abstract}
The most common sequelae in head and neck cancer patients submitted to radiotherapy (60C obalt) is postirradiation hyposalivation. This leads to a change in oral physiology, and, as a consequence, the irradiated individual is suceptible to infections and caries lesions. The aim of this study was to evaluate the behaviour of stimulated whole saliva flow rate (SW SFR) during and after radiotherapy treatment for head and neck cancer by lateral opposed fields. Six samples of mechanically stimulated whole saliva were collected of each individual and evaluated by gravimetric method. The first sample was collected before radiotherapy; the second one after 1500cG y dose; the third one after the last dose of treatment and the remainder samples in intervals of subsequent two months up to six months after the end of treatment. The SW SFR decreased in $50 \%$ after a dose of 1500cG y (1/4 of received treatment). After radiation therapy, the SW SFR was $0,33 \mathrm{ml} / \mathrm{min}$ and after six months was $0,20 \mathrm{ml} / \mathrm{min}$. These values represented, respectively, a decreasing in $64 \%$ and $78 \%$ of initial values (AN OVA, Tukey's Test $p \varangle 0.05$ ). Based in these results, the authors concluded radiotherapy when applied in malign neoplasms of the head and neck region by lateral opposed fields was able to decrease significantly salivary flow rate. Intensive preventive dental care during and after radiotherapy seems to be an useful tool to avoid radiation caries and their complications.
\end{abstract}

Key words: $\mathrm{H}$ ead and neck neoplasms; Xerostomia; $\mathrm{pH}$; Saliva.

\section{INTRO DUÇÃO}

A radioterapia é a modalidade terapêutica que utiliza as radiações ionizantes com o objetivo de destruir as células neoplásicas visando uma redução ou desaparecimento da neoplasia maligna. Esta modalidade, quando aplicada na região de cabeça e pescoço, dependendo da dose de irradiação, tempo de tratamento, volume do tratamento, dose de distribuição e do uso concomitante de outras terapias, pode produzir alterações reversíveis e irreversíveis nos tecidos. 0 s efeitos deletérios causados pela radioterapia considerados nesta região são aqueles que ocorrem nas glândulas sal ivares, 0ssos, dentes, mucosas da boca, músculos e articulações que combinam a perda de células e 0 dano na vascularização local ${ }^{1-3}$.

Atualmente, já está praticamente comprovado que doses de radiação entre 1000 e 1500cG y de C 060 são capazes de danificar de tal forma o funcionamento da glândula salivar humana, que o indivíduo queixa-se de xerostomia já no início da segunda semana de um protocolo convencional de tratamento ${ }^{4}$. Em animais de laboratório, o efeito da dose ainda parece inconclusivo. U ma simples dose de 250cG y já é suficiente para provocar al terações significativas na fisiologia das glândulas sal ivares destes animais ${ }^{5}$, enquanto que, uma dose de 4000cGy induz danos irreversíveis às células secretoras ${ }^{6}$.

A hipossalivação é uma das seqüelas mais freqüentes e é definida como uma condição clínica caracterizada pela redução qualitativa e quantitativa do fluxo salivar. Q uando a radioterapia é aplicada sobre as glândulas salivares e principalmente se a parótida estiver incluída na área irradiada, observa-se quea produção de saliva tornase comprometida. A hipossalivação quando pronunciada ocasiona danos na fisiologia bucal, tais como: dificuldade na mastigação, deglutição, fonação e um aumento na prevalência de infecções como a candidose, cárie e doença periodontal 7 . Tudo isto torna o indivíduo que foi irradiado um paciente de risco e que deverá ser submetido a um acompanhamento odontológico mais rigoroso.

O s relatos na literatura científica sobre as alterações quantitativas que ocorrem na saliva humana após a radioterapia de cabeça e pescoço, principalmente no Brasil, são escassos ${ }^{8}$. Vários estudos demonstraram que esta modalidade terapêutica empregada contra o câncer é capaz de induzir alterações significativas não somente no fluxo salivar, bem como, na concentração dos íons presentes na saliva ${ }^{9-17}$. O utro fato que permanece em discussão é saber se estas alterações salivares são temporárias ou definitivas. Sendo assim, realizamos este estudo objetivando descrever as possíveis alterações na velocidade do fluxo e pH da saliva total que ocorrem nos indivíduos portadores de câncer bucal e que receberam tratamento radioterápico padronizado na região de cabeça e pescoço.

\section{MATERIAL E MÉTO DOS}

Este estudo foi iniciado após seu protocolo ser submetido à apreciação e aprovação pela Comissão Científica da Faculdade de 0 dontologia e pelo Comitê de Ética em Pesquisa (CEP) do H ospital São Lucas da Pontifícia Universidade C atólica do R io Grande do Sul.

U m total de cento e quatro indivíduos adultos, dos gêneros masculino e feminino, com idade variando en- 
tre os 35 e 80 anos participaram deste estudo. Os indivíduos foram divididos em três grupos com um número mínimo de trinta pacientes em cada. 0 primeiro grupo (experimental) foi constituído por 42 pacientes portadores de neoplasia maligna na região da cavidade oral e/ou orofaringe submetidos a tratamento radioterápico. 0 segundo grupo, denominado grupo controle 01, constituiu-se de 32 indivíduos saudáveis, na mesma faixa etária do grupo anteriormente mencionado. 0 terceiro e último grupo foi denominado degrupo controle 02 , também constituído por indivíduos portadores de neoplasia maligna na região da cavidade bucal e/ou orofaringe, e que, não haviam sido submetidos a nenhuma modalidade terapêutica até aquelemomento. Estegrupo foi idealizado para descartar a possibilidade da própria neoplasia ser capaz de causar algum dano na fisiologia das glândulas salivares.

Antes que os indivíduos do grupo experimental iniciassem o tratamento radioterápico, o exame clínico da cavidade bucal foi realizado além de obtida uma amostra de saliva total. U ma coleta de saliva total estimulada foi obtida durante o curso do tratamento radioterápico (dose de $1500 \mathrm{cG}$ y) e mais quatro coletas subseqüentes, uma vez terminados o tratamento, totalizando assim, cinco coletas. Com o término do tratamento radioterápico, efetuou-se a primeira coleta desaliva total pós-tratamento eas três outras coletas subseqüentes, a cada dois meses, totalizando-se, desta maneira, um período de observação de seis meses pós-radioterapia.

Cada paciente do grupo experimental foi submetido a tratamento radioterápico pela técnica dos campos paral elos, opostos e laterais, cuja área irradiada incluiu, além da região do tumor, os linfonodos cervicais e as glândulas salivares maiores. A dose de radiação total aplicada foi em torno dos 7000cGy, fracionada em doses de 180 a 200cG y/dia. O s aparelhos empregados no tratamento radioterápico eram unidades de teleterapia rotacionais por Cobalto 60 (d-fótons) com energia de 1,25 M eV.

As amostras de saliva total foram obtidas através do método de coleta Spitting ${ }^{18}$ e o fluxo salivar estimulado mecanicamente pela mastigação de um pedaço de látex.
A velocidade do fluxo salivar foi obtida através do método gravimétrico ${ }^{19}$ e avaliadas segundo os critérios de KRASSE (1988) ${ }^{20}$. 0 pH salivar foi determinado pelo emprego dos indicadores vermelho de metila e azul de bromotimol, que permitem a diferenciação de valores de meia unidade entre 5 e 9.

\section{RESULTADO S}

A tabela 1 apresenta os valores da média e desviopadrão da variável velocidade do fluxo salivar total estimulada (VFSE) para o grupo experimental. Podemos observar neste grupo que a VFSE antes do tratamento encontrava-se dentro da faixa de normalidade segundo os critérios estabelecidos por KRASSE (1988) ${ }^{20}$, cuja média para esta variável foi de $0,92 \mathrm{ml} / \mathrm{min}$ para 0 período. Após o início do tratamento, os valores médios para a VFSE começaram a diminuir. Ao se atingir a dose de 1500 cG y, a média registrada foi de $0,46 \mathrm{ml} /$ min o que representa uma redução em cerca de $50 \%$ dos valores iniciais. N este momento, os pacientes já se queixavam de xerostomia e de uma dificuldade para a percepção do paladar. Clinicamente, pode-se observar que a saliva tornou-se mais espessa, ou melhor, com um aspecto mais mucoso. Segundo o relato dos pacientes era mais difícil de degluti-la e de comer os alimentos mais secos e sólidos. O utro achado diz respeito à coloração da saliva dos indivíduos do grupo experimental, que assumiu um aspecto visual mais amarelado. Ao término do tratamento, a VFSE encontrava-se mais reduzida, cuja média encontrada foi em torno de $0,33 \mathrm{ml} /$ min, tendo uma redução de $64 \%$ dos valores iniciais. $\mathrm{N}$ este momento, ao exame físico, a maioria dos pacientes apresentava as mucosas da cavidade bucal extremamente secas. D e acordo com o relato dos pacientes esta secura bucal causava incômodo principalmenteno período da noite. As amostras de saliva obtidas aos dois, quatro e seis meses após o término do tratamento radioterápico demonstraram que a VFSE continuava a diminuir gradativamente, cujas médias foram, respectivamente, $0,24 \mathrm{ml} / \mathrm{min}, 0,19 \mathrm{ml} / \mathrm{min}$ e $0,20 \mathrm{ml} / \mathrm{min}$. 0 que representou uma redução na VFSE

Tabela 1 - Média, desvio-padrão e coeficiente de variabilidade da velocidade do fluxo de saliva total estimulada em indivíduos submetidos à radioterapia na região da cabeça e pescoço. Curitiba/PR, 2004.

\begin{tabular}{|c|c|c|c|c|c|c|c|}
\hline $\begin{array}{l}\text { Velocidade do } \\
\text { fluxo salivar } \\
\text { estimulado } \\
\text { (ml/min) }\end{array}$ & $\begin{array}{c}\text { Pré } \\
\text { Radioterapia }\end{array}$ & $\begin{array}{c}\text { Durante } \\
\text { Radioterapia } \\
15 \mathrm{~Gy}\end{array}$ & $\begin{array}{l}\text { Pós } \\
\text { Radioterapia }\end{array}$ & $\begin{array}{c}2 \text { meses } \\
\text { Pós } \\
\text { Radioterapia }\end{array}$ & $\begin{array}{c}4 \text { meses } \\
\text { Pós } \\
\text { Radioterapia }\end{array}$ & $\begin{array}{c}6 \text { meses } \\
\text { Pós } \\
\text { Radioterapia }\end{array}$ & Valor de $p$ \\
\hline Média & 0,92 & 0,46 & 0,33 & 0,24 & 0,19 & 0,20 & 0,0000 \\
\hline Desvio-Padrão & 0,63 & 0,45 & 0,18 & 0,19 & 0,17 & 0,20 & - \\
\hline $\mathrm{CV}$ & 69,42 & 98,29 & 55,28 & 77,49 & 88,41 & 82,80 & - \\
\hline
\end{tabular}

Fonte: Serviço de Estomatologia do H ospital São Lucas da PU CRS, SERP e H ospital Santa Rita. 
destes indivíduos em torno de 74 a 79\%. N este grupo de indivíduos, 90,4\% queixaram-se de xerostomia e perda do paladar. Durante o acompanhamento clínico constatou-se que as mucosas permaneceram com aspecto visual bastante ressecado e atrófico, além de exibirem uma coloração mais avermelhada.

A tabela 2 demonstra a distribuição do número e percentual deindivíduos do grupo experimental segundo a VFSE de acordo com os critérios da Classificação de KRASSE (velocidade normal, baixa e hipossalivação). $\mathrm{N}$ ela podemos observar que antes do início do tratamento radioterápico cerca de $40 \%$ dos indivíduos já apresentavam valores para VFSE num nível de xerostomia. À medida que o tratamento ocorreu, esta porcentagem elevou-segradativamenteatéatingir $97,3 \%$, mantendo-se neste nível por até seis meses depois.

Tabela 2 - Distribuição do número e percentual de indivíduos do grupo experimental segundo a classificação de KRASSE para a velocidade do fluxo salivar estimulado. Curitiba/PR, 2004.

\begin{tabular}{ccccccc}
\hline $\begin{array}{c}\text { VFSE } \\
(\mathrm{ml} / \mathrm{min})\end{array}$ & $\begin{array}{c}\text { Grupo } \\
\text { Experimental } \\
\text { Pré }\end{array}$ & $\begin{array}{c}\text { Grupo } \\
\text { Experimental } \\
1500 \mathrm{cGy}\end{array}$ & $\begin{array}{c}\text { Grupo } \\
\text { Experimental } \\
\text { Pós }\end{array}$ & $\begin{array}{c}\text { Grupo } \\
\text { Experimental } \\
2 \text { meses }\end{array}$ & $\begin{array}{c}\text { Grupo } \\
\text { Experimental } \\
4 \text { meses }\end{array}$ & $\begin{array}{c}\text { Grupo } \\
\text { Experimental } \\
6 \text { meses }\end{array}$ \\
\hline 0,7 & $17(40,5 \%)$ & $32(82,05 \%)$ & $35(97,3 \%)$ & $33(94,3 \%)$ & $35(97,3 \%)$ & $37(97,4 \%)$ \\
$\geq 0,7 \mathrm{e}<1$ & $11(26,2 \%)$ & $4(10,25 \%)$ & $1(2,7 \%)$ & $2(5,7 \%)$ & $1(2,7 \%)$ & $1(2,6 \%)$ \\
$\geq 1$ & $14(33,3 \%)$ & $3(7,7 \%)$ & $0(0 \%)$ & $0(0 \%)$ & $0(0 \%)$ & $0(0 \%)$ \\
Total & $42(100 \%)$ & $39(100 \%)$ & $36(100 \%)$ & $35(100 \%)$ & $36(100 \%)$ & $38(100 \%)$ \\
\hline
\end{tabular}

Fonte: Serviço de Estomatologia do H ospital São Lucas da PUCRS, SERP e H ospital Santa Rita.

PH SALIVAR

$\mathrm{O} \mathrm{pH}$ da saliva dos indivíduos do grupo experimental sofreu uma redução significativa em função do tratamento empregado como podemos verificar através da tabela 3. Esta redução pouco ultrapassou a faixa de valores normais para esta variável, cujos valores considerados normais estão entre 6,1 e 8,0. Verificouse neste grupo, valores abaixo dosíndices normais apenas em dois indivíduos que exibiram $\mathrm{pH}$ salivar de 5,5. Os valores abaixo de 5,5 são considerados como pH crítico para que ocorra a desmineralização do esmalte dentário e tenham início as lesões de cárie que são freqüentemente observadas em pacientes irradiados.
0 grupo experimental exibiu valor médio de 7,6 antes da radioterapia, no entanto, este valor diminuiu para 6,8 logo após receber a dose equivalente a $1500 \mathrm{cG}$ y, mantendo-se neste nível por até 2 meses após o término do tratamento. Q uatro meses depois do tratamento 0 $\mathrm{pH}$ diminuiu ainda mais, alcançando o valor médio de 6,6 e novamente retornando a 6,8 quando esta variável foi avaliada seis meses após o término do tratamento. $\mathrm{A}$ análise de variância e o teste de Tukey determinaram que esta redução nos valores do pH salivar em função do tratamento radioterápico é estatisticamente significativa para $p<0,05$ (tabela 3).

Tabela 3 - Média e desvio-padrão do $\mathrm{pH}$ da saliva total estimulada em indivíduos submetidos à radioterapia na região da cabeça e pescoço. Curitiba/PR, 2004.

\begin{tabular}{cccccccc}
\hline pH & $\begin{array}{c}\text { Grupo } \\
\text { Experimental } \\
\text { Pré }\end{array}$ & $\begin{array}{c}\text { Grupo } \\
\text { Experimental } \\
1500 \text { cGy }\end{array}$ & $\begin{array}{c}\text { Grupo } \\
\text { Experimental }\end{array}$ & $\begin{array}{c}\text { Grupo } \\
\text { Experimental } \\
2 \text { meses }\end{array}$ & $\begin{array}{c}\text { Grupo } \\
\text { Experimental } \\
4 \text { meses }\end{array}$ & $\begin{array}{c}\text { Grupo } \\
\text { Experimental } \\
6 \text { meses }\end{array}$ & $\begin{array}{c}\text { Valor de } p \\
\text { Média }\end{array}$ \\
7,6 & $6,8^{*}$ & $6,8^{*}$ & $6,8^{*}$ & $6,6^{*}$ & $6,8^{*}$ & 0,0000 \\
$\begin{array}{c}\text { Desvio- } \\
\text { Padrão }\end{array}$ & 0,63 & 0,8 & 0,8 & 0,8 & 0,6 & 0,5 & 7,76 \\
CV & 8,22 & 11,68 & 11,83 & 11,74 & 10,45 & & 7,6 \\
\hline
\end{tabular}

* Diferença estatística (AN OVA, Teste de Tukey para comparações múltiplas $p \varangle 0,05$ ).

Fonte: Serviço de Estomatologia do H ospital São Lucas da PUCRS, SERP e H ospital Santa Rita. 


\section{DISCUSSÃO}

Estudos tentam estimar a freqüência com que as manifestações estomatológicas ocorrem nos indivíduos submetidos às terapias contra o câncer. A hipossalivação figura com uma freqüência de queixa em torno de $53 \%$, $60 \%$ e $100 \% 4,11,21$. N o nosso estudo, trinta e oito dos quarenta e dois indivíduos avaliados, queixaram-se de uma sensação de boca seca já a partir da segunda semana do tratamento radioterápico, representando, desta maneira, um percentual de 90,4\%. Esta discrepância entre os resultados observados neste trabalho quando comparados com os resultados da literatura podem ser em decorrência das diferentes metodologias empregadas.

Os resultados deste experimento comprovaram que uma dose de $1500 \mathrm{cG}$ y, que corresponde a oitava ou nona sessão do tratamento radioterápico, já é suficiente para reduzir a velocidade do fluxo salivar em cerca de $50 \%$. Estes achados estão em concordância com os valores encontrados na saliva total humana dos estudos realizados por DREIZEN et al. ${ }^{9}$; KUTEN et al. ${ }^{11}$; BU CHER et al. ${ }^{12}$. M esmo com o término do tratamento radioterápico, a velocidade do fluxo salivar do grupo experimental continuou a diminuir de forma gradativa por até seis meses. 0 s achados encontrados na literatura para esta variável demonstraram uma certa divergência. BERN H OFT e SKAUG 10 observaram um decréscimo ainda maior no fluxo salivar ao final da radioterapia e trinta dias depois, cujas médias obtidas foram de 0,07 $(-81 \%)$ e $0,04 \mathrm{ml} / \mathrm{min}(-89 \%)$, respectivamente. MAKKONEN ${ }^{2}$ percebeu reduções no fluxo salivar em torno de $71 \%$ após o tratamento radioterápico e de $92,8 \%$ seis meses depois. Já LIU et al. ${ }^{13}$ encontraram ao final, um mês e meio e seis meses após tratamento radioterápico, uma redução no fluxo salivar na ordem de $81 \%, 83,3 \%$ e $93,4 \%$. Estes achados demonstram que o dano induzido pela irradiação ao parênquima da glândula salivar persiste, mesmo que a radioterapia tenha sido sustada. No entanto, não podemos deixar de acreditar que outros fatores possam participar como coadjuvantes neste efeito de redução da quantidade de saliva dos pacientes irradiados. Conforme afirmaram THIEL et al. ${ }^{21}$, os pacientes portadores de neoplasias malignas, em geral, são indivíduos de idade avançada, usuários de diversos tipos de medicações e que expressam uma alteração no seu estado nutricional. Todos estes fatores podem contribuir para reduzir o fluxo salivar no indivíduo. Ao final do tratamento, quando os pacientes receberam em média uma dose final de 6800 cG y, a média para a velocidade do fluxo salivar estimulado encontrou-se ainda mais reduzida, pois se registrou um decréscimo na ordem de $79 \%$ quepersistiu pelo período de seis meses de pós-tratamento. LIU et al. ${ }^{13}$ e FUNEGARD et al. ${ }^{15}$ ao avaliarem o fluxo salivar de indivíduos que foram irradiados através de campos laterais, paralelos e oposto, notaram que há uma diminuição significativa na produção da saliva, podendo em alguns casos, ocorrer a ausência total de produção desta secreção. Tal fato acontece em virtude do dano irreversível provocado pelas doses empregadas no tratamento, que, geralmente variaram entre $6000 \mathrm{e}$ $7000 \mathrm{cG}$ y. De acordo com VISSINK et al. ${ }^{6}$, as doses acima de 4000 cG y já são suficientes para ocasionar profundas alterações no parênquima glandular, seguido por sua atrofia e fibrose.

$\mathrm{N}$ a distribuição do número de indivíduos do grupo experimental, segundo os valores registrados para a velocidade do fluxo salivar sob os critérios da classificação de KRASSE ${ }^{20}$, notou-se que antes do início do tratamento radioterápico, somente $40 \%$ dos indivíduos apresentavam valores acima de $1 \mathrm{ml} / \mathrm{min}$. Segundo MIRA et al. ${ }^{1}$, indivíduos que possuem um fluxo salivar elevado antes de iniciarem a radioterapia, coincidentemente, apresentarão uma menor redução no seu fluxo salivar. D 'H ON DT et al. ${ }^{17}$ ao acompanharem 34 indivíduos sob tratamento radioterápico discordaram desta teoria. Ao final do tratamento radioterápico e até seis meses depois, nenhum paciente deste estudo apresentou a velocidade do fluxo salivar em valores considerados na faixa de normalidade. Cerca de $97 \%$ destes estavam incluídos na categoria de hipossalivação, ou seja, com uma média de velocidade do fluxo salivar em torno de $0,20 \mathrm{ml} / \mathrm{min}$. Sendo assim, os achados deste estudo corroboram com D'HONDT et al. ${ }^{17}$ reforçam que não há relação de um menor dano ao funcionamento do parênquima glandular naqueles indivíduos que apresentavam um fluxo salivar elevado antes do tratamento radioterápico.

Alterações significativas nos valores do pH após os indivíduos receberem a dose de 1500 cG y também foram evidentes neste trabalho. $\mathrm{O} \mathrm{pH}$ da saliva está diretamente relacionado com a concentração de 
bicarbonato salivar, de tal forma que, à medida que 0 fluxo salivar aumenta, a concentração deste íon também se eleva e o reflexo é um $\mathrm{pH}$ salivar mais alto ${ }^{22} . \mathrm{Na}$ saliva não estimulada, o pH é em torno de 7,8 . Entretanto, valoresmais el evados podem ser encontrados a medida em que a saliva entra em contato com a atmosfera, através da perda de $\mathrm{CO}_{2}$. LESLIE eD ISCHE E ${ }^{16}$ observaram que $0 \mathrm{pH}$ da saliva estimulada de glândulas parótidas irradiadas diminuíram de 7,4 para 6,98. A análise do $\mathrm{pH}$ do grupo experimental deste estudo demonstrou um comportamento semelhante para esta variável, pois a média registrada antes do tratamento radioterápico foi de 7,6, baixando para 6,89 após os indivíduos receberem a dose de 1500cG y e manteve-se neste nível por até seis meses depois. O bservou-se apenas dois indivíduos do grupo experimental com valores do $\mathrm{pH}$ salivar dentro da chamada faixa de "pH crítico". N o entanto, seis meses após a radioterapia nenhum paciente apresentou valores abaixo de 6,0.

Com base nos resultados obtidos pode-se considerar que a radioterapia por $\mathrm{C} 060$ uma vez empregada no combate aos tumores malignos sediados na região da cabeça e pescoço é capaz de induzir alterações significativas sobre a velocidade do fluxo e pH da saliva total humana a partir da dose de 1500 cGy. Estas alterações ocorridas poderão ser de caráter transitório ou permanecerem por mais de seis meses. Além disso, descartamos a possibilidade de um tumor sediado na cavidade bucal, desde que não esteja envolvendo as glândulas salivares maiores, ser capaz de comprometer a fisiologia destas glândulas.

\section{CONCLUSÃO}

A Radioterapia por Cobalto60 quando usada na região da cabeça e pescoço é capaz de provocar uma redução significativa e progressiva no pH ena velocidade do fluxo de saliva total estimulada $(p \varangle 0,05)$.

\section{AG RADECIMENTO S}

Ao Conselho $\mathrm{N}$ acional de $\mathrm{D}$ esenvolvimento Científico e Tecnológico (CN Pq), pela concessão de bolsa de D outorado ao autor Antonio Adilson Soares de Lima. Ao Laboratório de Bioquímica e ao SERP Serviço de Radioterapia - do H ospital São Lucas da PU CRS e ao Serviço de Radioterapia do H ospital Santa
Rita no C omplexo H ospitalar I rmandade da Santa Casa de M isericórdia de Porto Alegre.

\section{REFERÊNCIAS}

1. M iraJG, Fullerton GD, WescottW B. Correlation between initial sal ivary flow rateand radiation dosein the production of xerostomia. ActaRadiol O ncol. 1982;21(3):151-4.

2. M akkonen TA. Studies on oral complication sof head and neck cancer radiotherapy. Proc Fin D ental Soc. 1988;84(45):1-111.

3. Jansma J 0 ral sequelae resulting from head and neck radiotherapy. C ourse, prevention and management of radiation caries and others complications [thesis]. G roningen: Riksuniversiteit G roningen; 1991.

4. M akkonen TA, N ordman E. Estimation of long-term salivary gland damage induced by radiotherapy. Acta $\mathrm{O} \mathrm{ncol}$. 1987;26:307-12.

5. N agler RM , Baum BJ, M iller G. Long-term salivary effects of single-dose head and neck irradiation in the rat. Archs O ral Biol. 1998;43:297-303.

6. Vissink A, Panders AK, Gravenmade J, Vermey A. The causes and consequences of hyposal ivation. Ear N oseT hroat J. 1988;67:166-76.

7. Atkinson JC, W U AJ. Salivary gland dysfunction: causes, symptoms and treatment. J Am Dent Assoc. 1994;125:409-16.

8. M atosIS, SilvaI L, Agra CM . Avaliação do fluxo salivar em pacientes submetidos a radioterapia de cabeça e pescoço. Rev Fac O dont U FBA. 1994/5;14:6-10.

9. D reizen $S$, Brown $L R, H$ andler $S$. Radiation-induced xerostomia in cancer patients. Effects on salivary and serum electrolytes. Cancer. 1976;38:273-8.

10. Bernhoft CH , Skaug N . O ral findingsin irradiated edentulous patients. Int J O ral Surg. 1985;14:416-27.

11. Kuten A, Ben-Aryeh H, Berdicevsky I. O ral side effects of head and neck irradiation: correlation between clinical manifestationsand laboratory data. Int J Radiat O ncol Biol Phys. 1986;12(12):401-5.

12. Bucher JA, Fleming TJ, Fuller LM . Preliminary observa tions on theeffect of mantle field radiotherapy on salivary gland flow rates in patients with $\mathrm{H}$ odgkin's disease. J D ent Res. 1988;67(2):518-21.

13. Liu RP, FlemingTJ, Toth BB. Salivary flow ratesin patients with head and neck cancer 0.5 an 25 years after radiotherapy. O ral Surg O ral M ed O ral Pathol. 1990;70(6):724-9.

14. Franzén L, Funegard U, Ericson TH . Parotid gland function during and following radiotherapy of mal ignancies in thehead and neck. A consecutivestudy of salivary flow and patient discomfort. Eur J Cancer. 1992;28(2/3):457-62.

15. Funegard U, Franzén L, Ericson TH . Parotid saliva composition during and after irradiation of head and 
neck cancer. O ral O ncol. 1994;30B(4):230-3.

16. Leslie M D , D ischeS. The early changes in salivary gland function during and after radiotherapy given for head and neck cancer. Radiother O ncol. 1994;30:26-32.

17. D 'H ondt E, Eisbruch A, Ship JA. The influence of preradiation sal ivary flow rates and radiation dose on parotid salivary gland dysfunction in patients receiving radiotherapy for head and neck cancers. Spec C are Dent. 1998;18(3):102-8.

18. N avazesh M . M ethod for collecting saliva. In: Saliva as a diagnostic fluid. Ann N Y Acad Sci. 1993;694:72-7.

19. Banderas Tarabay JA, Gonzáles-Begne M, Sánchez-
Garduño M , M illan CortezE, Lopez RodríguesA, Vilchis Velazquez A. Flujo y concentración de proteínas en saliva total humana. Salud Publica M ex. 1997;39:433-41.

20. K rasse B. Risco de cárie: um guia prático para avaliação e controle. 2a ed. São Paulo: Q uintessenceBooks; 1988.

21. Thiel HJ, Fietkau R, Sauer R. M alnutrition and role of nutritional support for radiation therapy patients. Recent Results C ancer Res. 1988;108:205-26.

22. Suddick RP, Hyde RJ, Feller RP. Saúde bucal, água e eletrólitossalivares. In: $M$ enaker $L, M$ orhart RE, $N$ aviaJ M . Cáriesdentárias: bases biológicas. Rio deJ aneiro: Guanabara Koogan; 1984. p. 118-31. 